\title{
VLT/ISAAC $H$-band spectroscopy of embedded massive YSOs
}

\author{
T. R. Kendall ${ }^{1}$, W. J. de Wit ${ }^{2}$, and J. L. Yun ${ }^{1}$ \\ 1 Centro de Astronomia e Astrofísica da Universidade de Lisboa, Observatório Astronómico de Lisboa, Tapada da Ajuda, \\ 1349-018 Lisboa, Portugal \\ 2 Osservatorio Astrofisico di Arcetri, Largo E. Fermi 5, 50125 Florence, Italy \\ e-mail: dewit@arcetri.astro.it
}

Received 2 December 2002 / Accepted 16 April 2003

\begin{abstract}
We have performed intermediate resolution $(R \sim 5000)$, high signal-to-noise $H$-band spectroscopy of a small, initial sample of three massive embedded young stellar objects (YSOs), using VLT/ISAAC. The sample has been selected from sources characterised in previous literature as being likely of OB spectral type, to be unambiguously associated with bright $(H \leq 14)$ single point sources in the 2MASS database, and to have no optical counterparts. Of the targets observed, one object shows a $\sim$ B3 spectrum, similar to a main sequence object of the same spectral type. A second object exhibits weak He I and $\mathrm{H}$ emission, indicating an early-type source: we detect He II absorption, which supports a previous indirect derivation of the spectral type as mid-O. The third object does not show absorption lines, so no spectral type can de derived. It does, however, exhibit a rich spectrum of strong, broad emission lines and is likely to be surrounded by dense circumstellar material and at a very early evolutionary stage. Our results from this very small sample are in agreement with those of Kaper et al. (2002), who also find spectra similar to optically visible main sequence stars, together with emission line objects representing a very early evolutionary phase, in their much larger sample of $K$-band spectra.
\end{abstract}

Key words. stars: formation - stars: early-type - ISM: H II regions

\section{Introduction}

Little is known about the earliest stages of the formation of massive stars. It is unclear whether the general picture applicable to the formation of low-mass stars, i.e. an accretion disk and infall onto the central star (Shu et al. 1987) can be scaled up to masses higher than $\sim 10 M_{\odot}$ (in the case of spherically symmetric accretion), as radiation pressure from the high mass stellar core on dust mixed with the infalling gas prevents further accretion. Alternative explanations involving the coalescence of intermediate mass protostars in the dense central regions of rich clusters have been proposed (Bonnell et al. 1998).

Observationally, the earliest stages of massive star formation are only accessible by infrared and radio techniques, because of the presence of $\sim 10-100$ mag of visual extinction. Ultracompact (UC) HII regions represent this earliest phase in the evolution of a very massive star. UC H II regions are typically identified using radio surveys (e.g. Bronfman et al. 1996; Walsh et al. 1999). Massive YSOs capable of ionizing a UC H II region are recognisable by their extremely red IRAS colours (Wood \& Churchwell 1989; Osterloh et al. 1997), and the IRAS database has become a rich source to be mined for intermediate and high-mass YSOs using colour selection criteria

Send offprint requests to: T. R. Kendall, e-mail: tkendall@oal.ul.pt (e.g. Persson \& Campbell 1987; Wood \& Churchwell 1989; Campbell et al. 1989; Chan et al. 1996).

Despite this, measurements of the fundamental parameters of UC H II regions, and more importantly those of the massive embedded stars which ionize the region, cannot easily be derived from the radio and far-IR observations. This is due principally to uncertainties in distances and in the indirect methods used to convert radio continuum or IRAS fluxes to the physical properties of the embedded, ionizing OB star (Walsh et al. 1997; Kaper et al. 2002 and references therein).

To circumvent these difficulties, observational efforts are now beginning to probe directly the photospheres of massive embedded YSOs using observations in the near-infrared. Such wavelengths are accessible from the ground (1-5 $\mu \mathrm{m}, J H K L M$ bands) and the extinction owing to the gas and dust in which the YSO is embedded is much less than in the optical. Moreover, at thermal infrared wavelengths, emission from the dust becomes too great, and the flux from the photosphere itself can no longer be detected. The first spectral classification of the ionizing star of a UC H II region was made by Watson \& Hanson (1997) in the $K$-band.

While an important aim of the present paper is to clarify the status of the three objects discussed here as massive embedded YSOs, a second no less important aim is to show that intermediate resolution spectroscopy in the $H$-band (where veiling from circumstellar emission is minimal) is a necessary and valuable 
tool to detect, classify and study the photospheres of such objects. This paper attempts to build on recent work on the classification of $\mathrm{OB}$ stars in the $H$-band, and to apply it to highly obscured objects.

\subsection{Classification spectroscopy of $O B$ stars in the $\mathrm{H}$-band}

Recent developments in classification spectroscopy of OB stars in the $H$ - and $K$-bands suggest application to objects too deeply obscured to be optically visible. In the earliest work, both Lancon et al. (1992) and Dallier et al. (1996) presented libraries of stellar spectra in the $H$-band, but these were largely directed to stellar population analyses. More recently, the most important study directly relevant here has been that of Hanson et al. (1998), who have identified a number of $\mathrm{H}$ band temperature and luminosity diagnostics for $\mathrm{OB}$ stars in $R=2000$ spectra, involving the ratio of $\mathrm{Br} 11(1.681 \mu \mathrm{m})$ to He I $(1.700 \mu \mathrm{m})$ and He II $(1.693 \mu \mathrm{m})$. Using a full grid of spectra representing both dwarf and supergiant luminosities, these authors find that the presence of all three lines definitively indicates a spectral type earlier than late $\mathrm{O}$. With combinations of only two lines present, in various strengths, dwarfs can be divided into O9-B1, B2-B5, and late B-early A type.

This work overlaps with that of Meyer et al. (1998), who have observed $85 \mathrm{MK}$ standards of near-solar metallicity, at $R \sim 3000$ in the $H$-band. They find a number of strong $T_{\text {eff }}$ and luminosity-sensitive lines in the $H$-band spectra of stars of spectral type A, and later, all the way down to M5. These two studies show that the classification of heavily embedded YSOs, and the near-IR study of their photospheres, could be carried out in the $H$-band for all spectral types from $\mathrm{O}$ to $\mathrm{M}$, using intermediate resolution, high signal-to-noise spectra. This could be advantageous, since the veiling of photospheric absorption lines by continuum emission from hot circumstellar dust is much less severe than in the $K$-band (Meyer et al. 1998; Ishii et al. 2001). Taken together, these studies suggest that while $K$-band spectroscopy has been extremely successful in studies of low-mass YSOs (see Greene \& Lada 1996 for one example among many) classification in the range B0-G0 is best performed in the $H$-band, as pointed out by Luhman \& Rieke (1998).

\section{Observations and data reduction}

\subsection{Target selection}

Targets for this study were selected from recent literature on UC H II regions and IRAS-selected catalogues of massive YSOs (see Table 1). The main criteria were that such targets would be sufficiently bright in the $H$-band to be accessible for medium resolution ISAAC spectroscopy in short exposure times, and that they should be present in the publicly available 2MASS dataset, to avoid the need for prior imaging. A further important criterion was that the 2MASS point sources should have no optical counterparts in the GSC2.2 and USNO catalogues. The three selected targets are listed in Table 1 together with 2MASS magnitudes and colours, $A_{\mathrm{v}}$, and indirect
Table 1. Basic and derived data for observed targets. Magnitudes and colours are taken from the 2MASS database, except for IRAS 171753544 by Tapia et al. (1996).

\begin{tabular}{lllllll}
\hline \hline IRAS & $H$ & $J-H$ & $H-K$ & $A_{\mathrm{v}}$ & Sp.T. & Int./s \\
\hline $17175-3544^{a}$ & 14.00 & 2.75 & 3.43 & $>13^{d}$ & O6-O7 & 7560 \\
$17441-2910^{b}$ & 10.29 & 3.42 & 2.41 & 30 & O5.5 & 480 \\
$18079-1756^{c}$ & 11.09 & 2.67 & 1.67 & 74 & B & 960 \\
\hline
\end{tabular}

${ }^{a}$ Walsh et al. (1999).

${ }^{b}$ Porter et al. (1998), Walsh et al. (1997).

${ }^{c}$ Osterloh et al. (1997).

${ }^{d}$ Using the given spectral type and using a distance of $2.2 \mathrm{kpc}$ (see text) we derive $m_{\mathrm{v}}=6$, yet there is no optical counterpart on DSS plates.

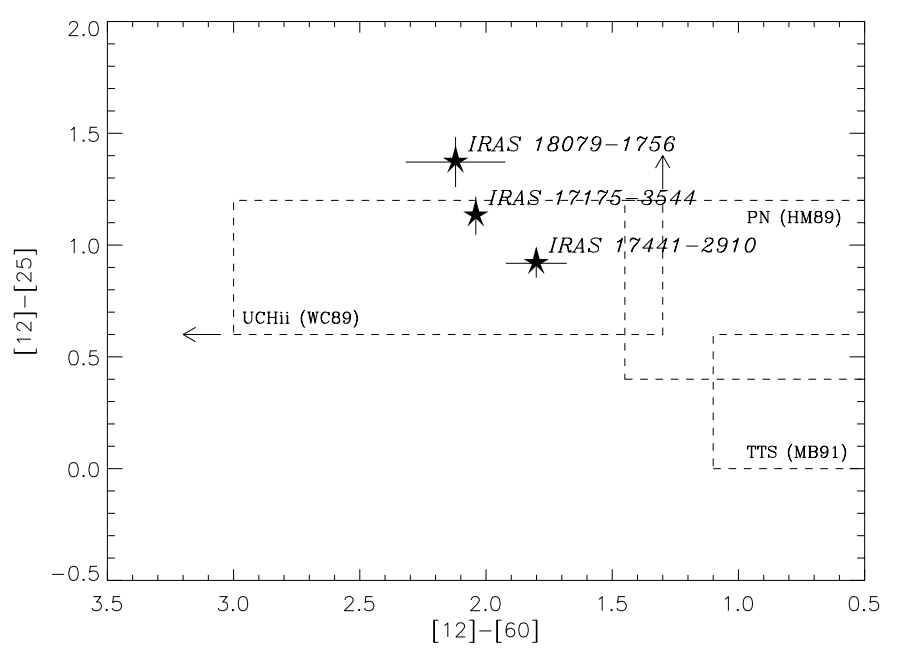

Fig. 1. IRAS colour-colour plot of the target objects as filled asterisk with errorbars, except the [12]-[60] error of IRAS 17175-3544, for which the $60 \mu \mathrm{m}$ IRAS measurement is of bad quality. Also indicated are the colours of 1 . UC H II regions compiled by Wood \& Churchwell (1989), 2. Planetary Nebulae (Hughes \& MacLeod 1989), 3. T Tauri stars (Morgan \& Bally 1991). Figure adapted from Osterloh et al. (1997).

spectral type estimates from the given references. The midinfrared fluxes are shown in the IRAS colour-colour diagram (Fig. 1), where different locations occupied by sources of various types are indicated. IRAS $17175-3544$ is too faint in the $J$-band for 2MASS to give an accurate magnitude in this band. However, this source shows a strong near infrared excess as shown in Fig. 2 where its $J H K$ colours were derived instead from the deeper imaging of Tapia et al. (1996).

Figure 3 shows the 2MASS $H$-band images, covering $152^{\prime \prime}$ square (the FOV of ISAAC) for each target. Figure 3 can be usefully compared to Fig. 19 of Walsh et al. (1999) for IRAS 17175-3544, who show a $K$-band image overlaid with the positions of methanol and $\mathrm{OH}$ maser sites and $8.64 \mathrm{GHz}$ radio continuum emission, which are shown to be coincident with the near-infrared source. Furthermore, $L$-band imaging of IRAS $17175-3544$ by the same authors indicates that the source is extremely bright and red $(L=3.8, K-L=4.7)$. In the cases of IRAS 17441-2910 and IRAS 18079-1756, 2MASS $H$ band data indicate that the bright sources shown in Fig. 3 lie 


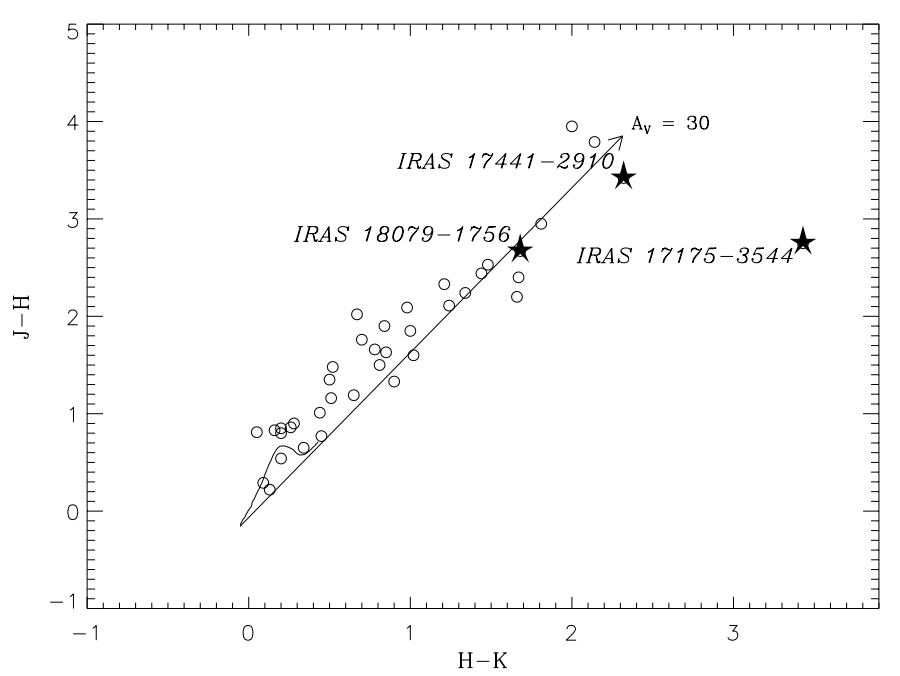

Fig. 2. 2MASS near infrared colour-colour diagram. Target object are indicated again by asterisks which are larger than the errorbars $( \pm 0.06 \mathrm{mag}$ or smaller for 2MASS colours, \pm 0.1 for Tapia et al. 1996). The short curve shows colours for main sequence stars (Koornneef 1983). The long straight arrow corresponds to displacements of an early type object suffering an extinction equal to $A_{\mathrm{V}}=30$. Displacement to the right of this line indicates a nearinfrared excess. The small open circles are 2MASS detected objects, within $1^{\prime}$ radius from the targets. Note the strong extinction towards our targets.

within $2-3^{\prime \prime}$ of the IRAS positions, allowing unambiguous identification of the targets.

The presence of nebulosity associated with all targets is clearly shown in the $H$-band images and even more obvious in the $K$-band (see Fig. 4, which uses the same intensity scales as the equivalent $H$-band images). This strengthens the identification of the sources in the 2MASS data with the IRAS sources given in the literature. The very bright source IRAS 174412910 appears to be multiple, but manipulation of the image indicates that there is one single, major source for which the point spread function in the 2MASS $H$-band image is symmetric. Therefore it was included as a target under the assumption that this one source would contribute by far the greater flux to the $H$-band spectrum.

\subsection{Data extraction}

Observations were carried out at VLT/UT1 (Antu) with the ISAAC spectrograph, in SW mode at medium resolution $(R \sim$ 5000), in service mode, on 2002 May 9 and 10. The total integration time for each object is given in Table 1 and totals $150 \mathrm{~min}$. A central wavelength of $1.71 \mu \mathrm{m}$ was selected to provide a wavelength range of $\sim 1.67-1.75 \mu \mathrm{m}$, covering lines diagnostic of an $\mathrm{OB}$ spectral type, most importantly H I Br $101.7362 \mu \mathrm{m}$, Br $111.6811 \mu \mathrm{m}$, He I $1.7007 \mu \mathrm{m}$ and He II $1.693 \mu \mathrm{m}$. Data reduction was performed with the ISAAC pipeline package ECLIPSE (version 4.1.2). Firstly, all data and calibration frames were "de-ghosted" using the ghost routine. Next, master flat fields were created for each night using the sw_spflat routine. Co-added spectrum images were then

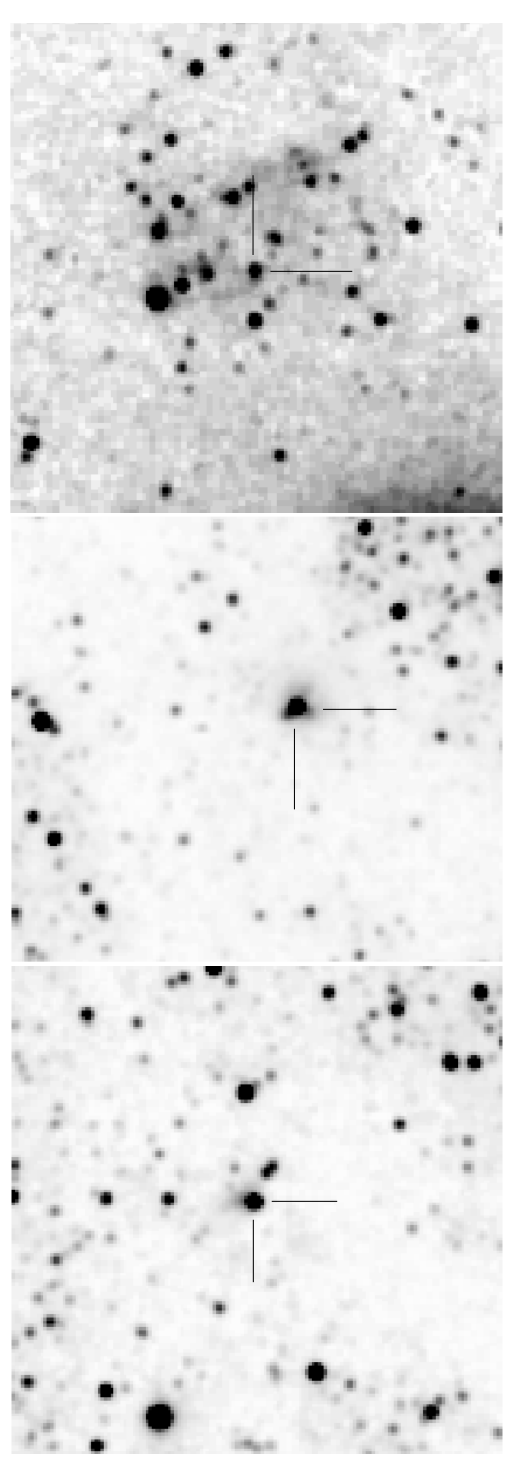

Fig. 3. 2MASS $H$-band image of IRAS 17175-3544 (top), IRAS 17441-2910 (centre) and IRAS 18079-1756 (bottom). North is up, East to the left. The field size is $2.5^{\prime}$ square.

created using the $s w$ spjitter routine. Full details of the ISAAC reduction pipeline can be found in Amico et al. (2002).

Spectra were then extracted using the standard APALL routine in $\mathrm{IRAF}^{1}$, and wavelength calibrated using the sky $\mathrm{OH}$ emission lines found throughout the spectra. In all cases $\sim 17 \mathrm{OH}$ lines were used, yielding rms values on the fits to the dispersion of $<0.1 \AA$.

Telluric divisor stars were selected at the time the observations were made, and were typically observed to within 0.05 airmasses of the science objects. Telluric standards were reduced, extracted and wavelength calibrated in exactly the same way as the science data. As the divisor stars are all of spectral type $\mathrm{B}, \mathrm{Br} 11, \mathrm{Br} 10$ and He I profiles were estimated by eye and directly fitted out of the standard spectra, and pure

\footnotetext{
${ }^{1}$ IRAF is distributed by the National Optical Astronomy Observatories, which is operated by the Association of Universities for Research in Astronomy, Inc. (AURA) under cooperative agreement with the National Science Foundation.
} 


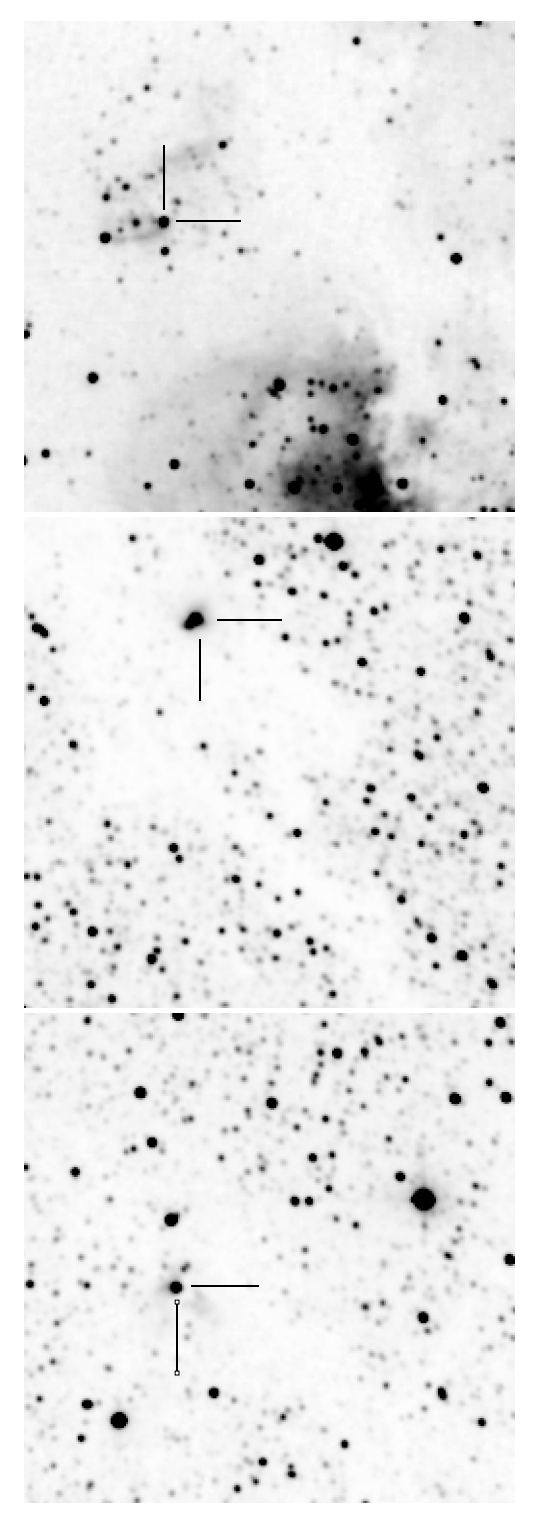

Fig. 4. 2MASS $K$-band image of IRAS 17175-3544 (top), IRAS 17441-2910 (centre) and IRAS 18079-1756 (bottom). North is up, East to the left. The field size is $4^{\prime}$ square. In the top panel (IRAS 17175-3544), the North-Eastern part of the image is NGC 6334 I; in the South-Western part is the star forming region NGC 6334 II (see text). Note the strong extinction, obvious from the small number of stars near the target objects, which are indicated.

telluric spectra created in these regions by dividing the spectrum by the fit. These sections were then spliced into the rest of the standard star spectra (assumed free of photospheric lines) to create normalised pure telluric spectra, one derived from each standard star observation.

One problem is that in all standard star spectra He I is itself blended with a telluric line, too closely for the components to be separated. Therefore the whole He I + telluric profile had to be fit, and replaced by a flat "continuum" region. Hence the region around He I remains uncorrected for telluric absorption in all target spectra shown in Fig. 5. The problem is dealt with further in Sect. 4, where we have used synthetic spectra to model the telluric standards (see Fig. 6).
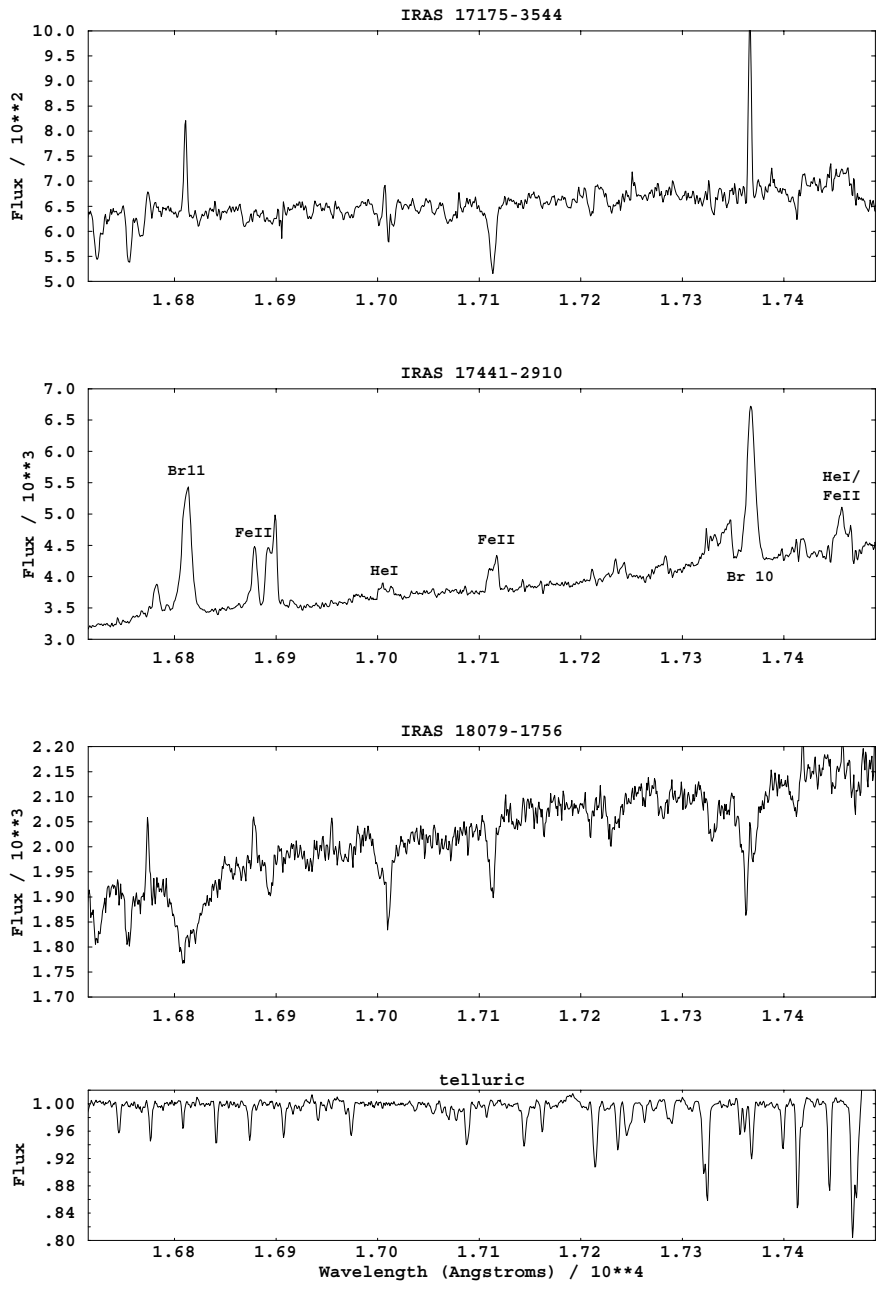

Fig. 5. $H$-band spectra of the target objects, covering the complete wavelength range 16715-17490 $\AA$, and corrected for telluric absorptions (except near $1.70 \mu \mathrm{m}$ : see text). Upper large panel: IRAS 171753544. Central: IRAS 17441-2910. Lower: IRAS 18079-1756. Note that the flux scale (counts) is different for IRAS 17441-2910, in order to show the strong emission lines. A normalised template telluric spectrum used is shown in the bottom smaller panel.

Object spectra were divided by those of the appropriate normalised standard (after fitting out photospheric lines) using the IRAF package TELLURIC. This method retains the same flux scale (number of counts) and spectral slope as the uncorrected spectra. The signal-to-noise ratio $(S / N)$ of the final spectra is $\geq 100$ and in the case of the very bright target IRAS 174412910, it approaches 150 .

\section{Background for individual objects}

The three target objects are located in heavily obscured regions in the Galactic plane. Strong extinction in the respective areas is especially obvious from 2MASS $K$-band images (Fig. 4). This results in a sharp drop in 2MASS $K$-band detections within a few arcminutes of the target objects. The obscuring material appears to have a filamentary structure, especially visible near IRAS 17441-2910 and IRAS 18079-1756. Neither of these two objects are located within a known massive star forming region or giant molecular cloud. 

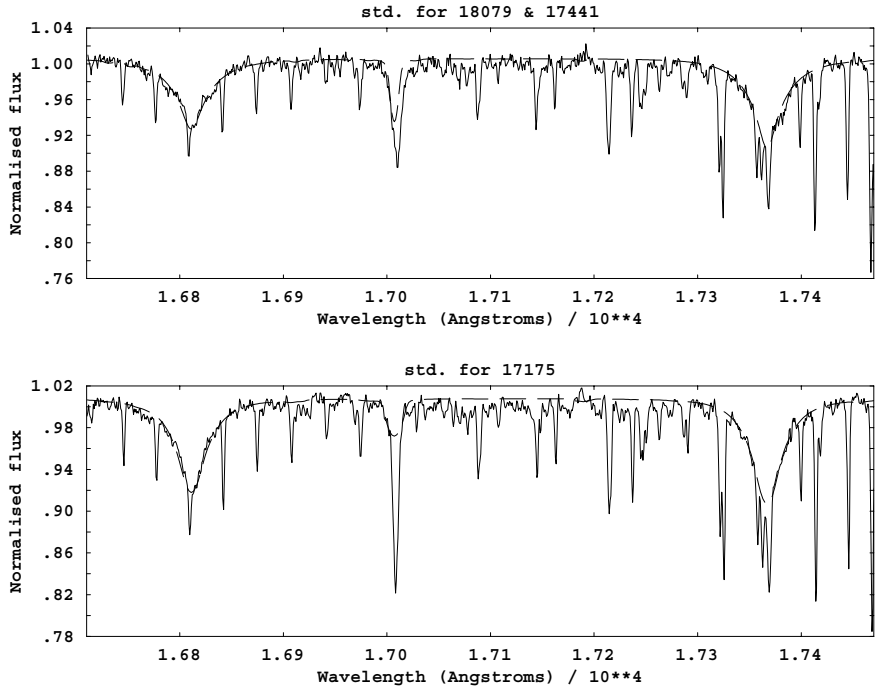

Fig. 6. Normalised spectra of telluric standards for IRAS 17175-3544 (top) and IRAS 18079-1756 (bottom) with best fit model spectra (dashed lines). See text for the model parameters.

By contrast, IRAS 17175-3544 lies in the North-Eastern part of the extended molecular cloud complex NGC 6334 (photometric distance $1.76 \mathrm{kpc}$, Neckel 1978). This star forming region is known as NGC 6334 I (Emerson et al. 1973; McBreen et al. 1979) and contains three IR sources IRS-I 1, IRS-I 2 and IRS-I 4 (Harvey \& Gatley 1983). IRS-I 1 and IRS-I 2 are within a few arcsec of each other, and both have been proposed to be the driving source of a high-velocity $\left(70 \mathrm{~km} \mathrm{~s}^{-1}\right)$ CO bipolar outflow (see Persi et al. 1996).

IRAS 17175-3544 itself appears to be associated with IRS-I 1 (see Fig. 19 of Walsh et al. 1999 and Persi et al. 1996). High resolution near-IR imaging showed IRS-I 1 to be complex, consisting of at least 4 components (Persi et al. 1996). The nearby $\left(6^{\prime \prime}\right)$ source IRS-I 2 is a mid-IR source (detected at 20 and $30 \mu \mathrm{m}$ ). Whether or not this source contributes to the $H$-band spectrum is determined by its extension and position with respect to the slit, but no source brighter than $K \sim 17.5$ is detected at the position of IRS-I 2 by Persi et al. (1996), so we consider such a contribution unlikely. This source could however contribute to the IRAS fluxes.

Further evidence for the massive YSO nature of at least two of the objects discussed in this work, namely IRAS 171753544 and IRAS $18079-1756$, is that both have IRAS low resolution $(8-23 \mu \mathrm{m})$ spectra classified as type $\mathrm{H}$ by Kwok et al. (1997). These authors state that the vast majority of IRAS LRS spectra of this type, exhibiting very red continua, are associated with $\mathrm{H}$ II regions.

Another noteworthy feature concerning IRAS 17175-3544 is that it is surrounded by an embedded stellar cluster (centred on IRS-I 2), which was detected in the $K$-band by Straw et al. (1989). The cluster is clearly visible in Fig. 4. Deeper imaging by Tapia et al. (1996) yielded a stellar density estimate of about $1200 \mathrm{pc}^{-3}$, and cluster size of $0.6 \mathrm{pc}$ (for $D=1.74 \mathrm{kpc}$ ).

In addition to the cluster near IRAS 17175-3544, our acquisition images revealed that IRAS $18079-1756$ is also accompanied by a embedded cluster, which the 2MASS image
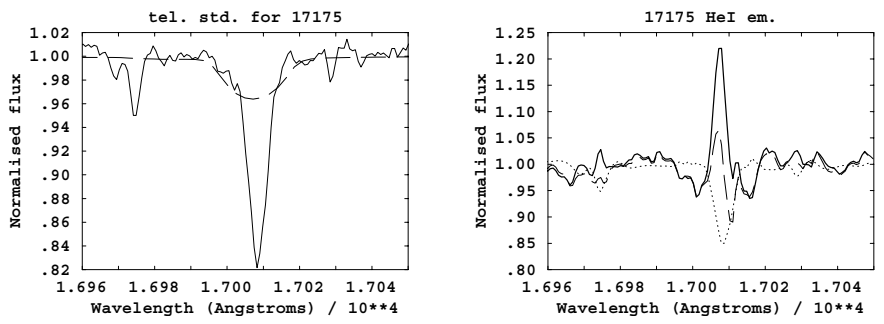

Fig. 7. Left panel: comparison of the region of the $\mathrm{He} \mathrm{I}+\oplus$ line in the telluric standard for IRAS 17175-3544 with the best-fit synthetic spectrum. (dashed line, see Fig. 6 for the complete synthesis). Right panel: the He I line in IRAS 17175-3544 (dashed line) together with a pure telluric line derived by division of the two spectra in the left panel (dotted line). The heavy line shows the results of the division, clearly showing the narrow He I emission in the IRAS 17175-3544 spectrum.

in Fig. 3 is too shallow to fully reveal. It is not clear whether there is an increase in stellar density near the third source IRAS 17441-2910.

Finally, apart from the location in an obscured region and the presence of a cluster (near at least two sources), a third common characteristic of the target sources is that all are associated with maser emission and/or are known to be outflow sources, as has already been mentioned for IRAS 17175-3544. IRAS 17441-2910 shows evidence for OH masering (Caswell 1998), while IRAS 18079-1756 displays $\mathrm{H}_{2} \mathrm{O}$ maser emission located at $\sim 12^{\prime \prime}$ distance and is known to be a $\mathrm{CO}$ outflow source (Osterloh et al. 1997). To conclude, all three sources show all the characteristics of young, actively forming massive stars.

\section{Results from individual spectra}

\subsection{IRAS 17175-3544}

Figure 5 shows the observed $H$-band spectrum of IRAS 17175 3544 (upper large panel). As has already been discussed, Walsh et al. (1999) find maser and radio continuum sources exactly coincident with the near-IR source discussed here. IRAS 17175-3544 is one of the few sources for which Walsh et al. (1999) can unambiguously derive a spectral type. As a result of a methanol maser emission survey to identify UC H II regions from an IRAS-selected sample, Walsh et al. (1997) calculated total luminosities and spectral types directly from the IRAS fluxes and the kinematic distances given by the maser emission. However, the large size of the IRAS beam $\left(\sim 1^{\prime}\right)$, may encompass a number of emitting regions, so the IRAS-based spectral types cannot be definitive.

In the case of IRAS 17175-3544, though, extrapolation of the near-IR SED to the far-IR shows that the near-IR counterpart will be the one dominant star in the field. Using a kinematic distance of $2.2 \mathrm{kpc}$, Walsh et al. (1999) find O6-O7 (from a total luminosity of $12.1 \times 10^{4} L_{\odot}$ ).

The $H$-band spectrum does not slope as steeply to the red as the other two objects in the sample, perhaps suggesting it is less embedded; yet the infrared excess is by far the largest of this sample. Narrow, relatively weak emission from $\mathrm{H}$ I $\mathrm{Br} 10$ and $\mathrm{Br} 11$ is clearly seen. In Fig. 5, the region around the He I 


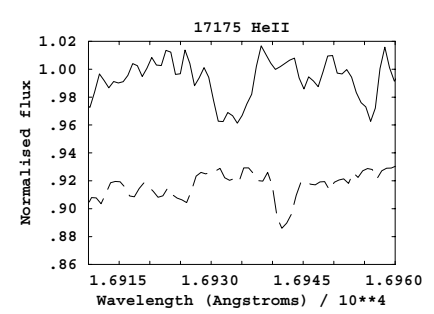

Fig. 8. Detection of the He II line at $\sim 1.693 \mu \mathrm{m}$ in absorption in IRAS 17175-3544. The telluric spectrum in the same region (offset for clarity) is also shown (dashed line) and suggests the He II detection is not a result of imperfect $\oplus$ cancellation.

line at $1.7007 \mu \mathrm{m}$ is not corrected for telluric lines, for reasons already discussed. However, there is certainly a suggestion of He I emission. This is clarified in Fig. 7, where in order to perform telluric correction in this region, we have fitted the complete telluric standard spectrum with a synthetic spectrum. For the standard for IRAS $17175-3544$, we adopt $T_{\text {eff }}=20000 \mathrm{~K}$, $\log g=3.5$ and $v \sin i=200 \mathrm{~km} \mathrm{~s}^{-1}$ (see also Fig. 6). The He I line for these parameters is shown in the left panel of Fig. 7 (dashed line) together with the blended He I + telluric profile in the standard star. After division, a pure telluric line is obtained (Fig. 7, right panel, dotted line). Using this profile, the He I region of IRAS 17175-3544 was corrected for telluric absorption, yielding a clear emission line rising to $\geq 20 \%$ above the continuum level. Using this line, together with the narrow HI emission, we derive an LSR radial velocity of $+15.9 \pm 1.5 \mathrm{~km} \mathrm{~s}^{-1}$ for this object. This is somewhat different from the methanol maser emission velocity given by Walsh et al. (1997) $\left(-10 \mathrm{~km} \mathrm{~s}^{-1}\right)$ and could indicate that the atomic and molecular emission do not arise in precisely the same circumstellar region. However we note that the velocity resolution of our data is insufficient to press this point. It is clear, however, from inspection of the 2D spectrum images for this object, that the $\mathrm{H}$ and $\mathrm{He}$ emission lines are extended spatially, and hence are of nebular origin.

By themselves, emission lines of $\mathrm{H}$ and He suggest an early-type object, but do not allow derivation of a spectral type. We may speculate that, as the emission is much weaker than in IRAS 17441-2910, either the exciting central object is not as luminous in this case, or perhaps the object is in an "intermediate" stage of evolution, displaying nebular emission but not the powerful circumstellar emission of IRAS 17441-2910, nor resembling a main sequence type spectrum like IRAS 180791756 (see below).

As this object has previously been characterised as O6-O7, we have searched the He II region carefully. Figure 8 shows this region in the telluric corrected spectrum, with the telluric standard star also plotted as a dashed line. The feature in the IRAS $17175-3544$ spectrum at $\sim 1.693 \mu$ m does not appear to be an artifact of the telluric correction process, since the standard star spectrum is featureless across its profile.

We identify this feature as He II absorption. The equivalent width is close to $300 \mathrm{~m} \AA$, compatible with the measurements of Hanson et al. (1998): in their small sample, they measure $0.5 \pm$ $0.2 \AA$ for their latest O-dwarf (HD 48279, O8V) and $0.3 \pm$ $0.2 \AA$ for the O7V HD 47839, while they do not detect He II in their $09.5 \mathrm{~V}$ object.
Moreover, Meyer et al. (1998) include the O7V star HR2546 (15 Mon) in their sample. Even their best spectrum of this object is rather noisy $(S / N \sim 50)$, but clearly exhibits He II absorption. Since the spectrum is noisy, and the continuum level around He II uncertain, we have not attempted to plot the profile in Fig. 8. However, the equivalent width is in the range $150-400 \mathrm{~m} \AA$, close to both Hanson et al. (1998) and our measurement. Our He II detection therefore supports the previous classification of IRAS 17175-3544 as O6-O7. A caveat, however, is that according to Hanson et al. (1998), He I should still be strong in absorption $(\sim 1 \AA)$ for an O7V type. Such absorption appears to be masked by nebular emission in IRAS 17175-3544.

That IRAS 17175-3544 is located at the centre of the complex star-forming region of NGC 6334 I has already been discussed. In addition to the $\mathrm{H}$ and $\mathrm{He}$ features already discussed, it shows absorption features which can be identified as $\operatorname{Mg} \mathrm{I}(17113 \AA)$ and the AlI triplet (16723, 16755 and $16768 \AA$ ). Usually these features are associated with cooler atmospheres, increasing in strength to K-types (Meyer et al. 1998). Inspection of the telluric standard spectrum excludes a telluric nature for this lines (unlike the emission spikes near Br 11 in IRAS 18079-1756), nor a badly determined and subtracted sky spectrum (contamination by other, late-type stars in the slit), since the slit position angles were carefully chosen to avoid any other stars along its length. However, a close examination of the VLT acquisition image for IRAS 171753544 reveals the presence of a second object, which would have been in the slit at the time the spectrum was obtained. The two sources are not resolved in the 2MASS image.

These two sources are coincident with IRS1-I1E and IRS-I 1W of Persi et al. (1996, see also Tapia et al. 1996) where the former source is shown by these authors to be the reddest ( $H=15, H-K=3.9$ ), dominating the emission longward of $2 \mu \mathrm{m}$ and coincident with the peak of the $30 \mu \mathrm{m}$ emission. This source is the OB star responsible for the ionization of the compact $\mathrm{H}$ II region and for pumping the nearby $\mathrm{H}_{2} \mathrm{O}$ and $\mathrm{OH}$ masers. The second source has $H=14.4$ and $J-H=2.3$, and clearly also contributes to the $H$-band spectrum, giving rise to the composite spectrum we observe. The presence of the $\mathrm{MgI}$ and $\mathrm{AlI}$ lines, presumed to arise from IRS1-I1W, shows that this source is cooler. We have performed experiments with synthetic spectra which show that the flux emitted by a $T_{\text {eff }}=20000 \mathrm{~K}$ photosphere is only $\sim 5$ times greater than one of $T_{\text {eff }}=5000 \mathrm{~K}$ in the $H$-band, so only a very few cooler embedded stars might suffice to cause the appearance of $\mathrm{Mg}$ I and $\mathrm{Al}$ I. In this scenario, IRS-I $1 \mathrm{~W}$ might be a system of less massive embedded objects associated with IRS-I 1E, the embedded $\mathrm{OB}$ star which gives rise to the $\mathrm{H}$ and $\mathrm{He}$ features in our spectrum.

Lastly, it might be argued that the observed spectrum could be that of a foreground late-type object, with emission lines from the nebular background. We cannot definitively rule out this possibility using the data presented here, but would point out that there is no optical counterpart to such a putative foreground object, even on the very deep SuperCosmos (UKST I-band, Hambly et al. 2001 and references therein) images. 


\subsection{IRAS 17441-2910}

The very high signal-to-noise $(\sim 150)$ spectrum of this bright $(H=10.29)$ source is shown in Fig. 5 (central large panel). The spectrum exhibits broad, strong emission lines of $\mathrm{H} \mathrm{I} \mathrm{Br}$ 10, $\mathrm{Br} 11$ and numerous other species, notably $\mathrm{He} \mathrm{I}$ and [Fe II]. The emission lines we have been able to identify are labelled in Fig. 5. The object is known to exhibit very strong CO emission in the $K$-band (Porter et al. 1998). The equivalent widths of the $\mathrm{Br} 11$ and $\mathrm{Br} 10$ emissions are $\sim 6 \AA$ and $\sim 5.5 \AA$ respectively, with $F W H M \sim 180 \mathrm{~km} \mathrm{~s}^{-1}$ and $160 \mathrm{~km} \mathrm{~s}^{-1}$. Porter et al. (1998) find $\mathrm{Br} \gamma$ in emission with equivalent width $12 \AA$. Walsh et al. (1997) have derived a spectral type of O5.5 from the IRAS fluxes, together with the kinematic distance given by the methanol maser $(6.67 \mathrm{GHz})$ emission profile $(9.8 \mathrm{kpc})$, and assuming the source is a single star. However, the lack of photospheric absorption lines precludes a spectral type determination here.

After correction to the LSR velocity, we derive a radial velocity of $+44.9 \pm 4 \mathrm{~km} \mathrm{~s}^{-1}$ using 4 lines labelled in Fig. 5 which we are able to deblend accurately: $\mathrm{Br} 11$ 16811.11, Br 10 17366.85, Fe II 16877.81 and Fe II $17115.95^{2}$. This velocity is somewhat different than that cited by Walsh et al. (1997), and again may indicate that the atomic emission arises in a different region from that producing the methanol maser emission (centred near $+23 \mathrm{~km} \mathrm{~s}^{-1}$ ). However, owing to the large $F W H M$ of the emission lines measured, and consequent difficulties in accurately measuring the line centre, we do not consider this point further.

Kaper et al. (2002) have drawn attention to a significant population of objects whose $K$-band spectra also show broad, strong emission lines, up to $100 \mathrm{~km} \mathrm{~s}^{-1}$, two of which also show CO emission. IRAS 17441-2910 clearly belongs in this category also. Following the suggestion of Kaper et al. (2002), IRAS 17441-2910 appears to be a third object for which strong emission of $\mathrm{CO}$ and other species, together with a significant infared excess (from 2MASS colours), indicate that the exciting object is surrounded by dense circumstellar material. It is clear that the strong emission line objects represent massive stars in the earliest stages of evolution.

\subsection{IRAS $18079-1756$}

Previous literature on this object gave $L_{\text {bol }} \sim 32000 L_{\odot}$ and $A_{\mathrm{v}}=74$ (Osterloh et al. 1997). This bolometric luminosity would correspond roughly to an early B spectral type. The entire $H$-band spectrum is shown in Fig. 5 (lower large panel). The plot shows that the continuum slope for IRAS 18079-1756 rises to the red across the length of the $H$-band spectrum by $\sim 20 \%$, comparable to IRAS 17441-2910, suggesting it is indeed deeply embedded. However, the absence of significant emission, and the general appearance of the spectrum (similar to an unobscured object of the same type) suggests that it could be at a somewhat later evolutionary phase.

The spectrum clearly shows broad He I absorption, which is centred at $\sim 17007 \AA$, as well as broad hydrogen

\footnotetext{
${ }^{2}$ Rest wavelengths from www. pa.uky. edu/ peter/atomic/.
}

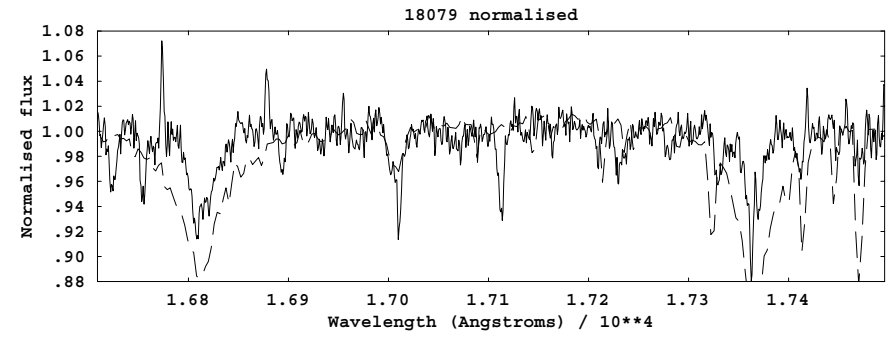

Fig. 9. Normalised spectrum of IRAS 18079-1756 overlain with the $H$-band spectrum (dashed line) of the fast rotator HR 5191 (spectral type B3V - see text). Good agreement in the region of the He I absorption can be seen. Mismatches in the strength of the $\mathrm{H}$ lines results from filling in of the line profile by emission.

absorption. In Fig. 9, the normalised spectrum of IRAS 180791756 is compared to an $H$-band spectrum of HR 5191 ( $~ \eta \mathrm{UMa}$ ). This star is one observed by Meyer et al. (1998), who give a spectral type of B3V and $v$ sin $i=205 \mathrm{~km} \mathrm{~s}^{-1}$. The He I profile is well matched, but the $\mathrm{H}$ lines are weaker than in the standard star, presumably because the $\mathrm{H}$ profiles are filled in by emission. This is supported by inspection of the $\mathrm{Br} 10$ profile, which clearly shows central emission. The He I profile is contaminated by a telluric line, since it was not possible to correct for telluric absorption over a $50 \AA$ region across the He I profile (see Sect. 2.2). Figure 10 (left panel, dashed line) shows the blended $\mathrm{He}+$ telluric profile in the B-type standard observed for IRAS 18079-1756. In the same way as for IRAS 17175-3544, we have synthesised this spectrum (see Fig. 6) adopting parameters of $T_{\text {eff }}=25000 \mathrm{~K}, \log g=4.0$ and $v \sin i=100 \mathrm{~km} \mathrm{~s}^{-1}$ (dotted line). Division yields a pure telluric profile (solid line). In Fig. 10 (right panel), this resultant profile (dotted line) is divided through the uncorrected spectrum for IRAS 18079-1756 (dashed line) to yield the corrected He I profile (heavy line). This profile matches the broad feature in Fig. 9 well, supporting the case that the stellar He I line is the broader feature. Comparing this profile to the galactic standard yields a very close match between the two profiles. It is clear that this match strongly supports an early-B spectral type for IRAS 18079-1756, and furthermore suggests it is a rapid rotator, as expected for very young massive YSOs. However, a rigorous measurement of $v \sin i$ would require spectra of rather higher resolution.

After telluric correction, the equivalent width of $\mathrm{He}$ I (heavy profile in Fig. 10) was measured as $600 \pm$ $100 \mathrm{~m} \AA$. Comparison with equivalent widths tabulated by Hanson et al. (1998) against optical spectral type, suggest IRAS $18079-1756$ is very close to B2, assuming a dwarf luminosity. Comparison with HR 5191 suggests B3V. However, strong He I persists in supergiants to $\sim$ B7. If IRAS 18079-1756 is of lower gravity, which would not be unexpected by analogy with low-mass embedded protostars (see Greene \& Lada 1996), then a somewhat later spectral type is possible. The equivalent width of $\mathrm{Br} 11$ is $\sim 2.0 \pm 0.1 \AA$, which also suggests early $\mathrm{B}$, although because of the possibility of emission this is not such a reliable indicator.

Furthermore, He I $1.700 \mu \mathrm{m}$ remains strong into the Ostar regime, as far as $\mathrm{O} 7$, and the $\mathrm{Br} 11$ widths given by 

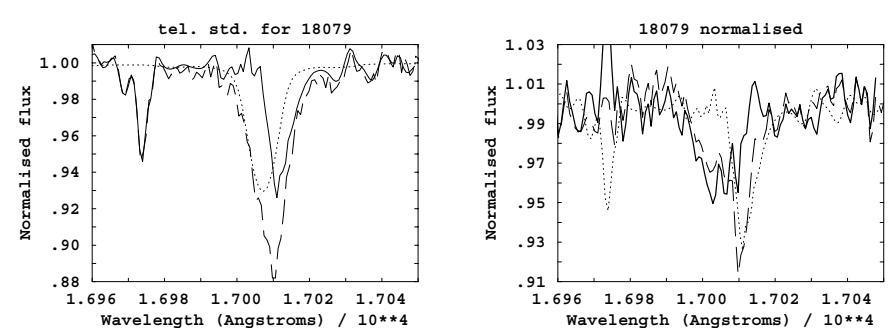

Fig. 10. Left panel: the blended $\mathrm{He} \mathrm{I}+\oplus$ profile in the telluric standard spectrum for IRAS 18079-1756 (dashed line) together with the adopted model He I line (dotted line; see text). The solid line shows the pure telluric line derived by division. Right panel: this pure telluric line (dotted) overlain with the uncorrected data for IRAS 18079-1756 (dashed line). The heavy line shows the corrected He I profile for this object.

Hanson et al. (1998) for their late O-stars show large scatter, varying non-monotonically from 1.6 to $3.0 \AA$ between $09.5 \mathrm{~V}$ and O7V. If IRAS 18079-1756 is as early as O8V, however, it should show He II absorption at $1.693 \mu \mathrm{m}$ with a strength comparable to that of the observed He I profile. Such a feature is not seen. Hanson et al. (1998) do not detect He II in their O9.5V object, so in principle IRAS $18079-1756$ could be as early as this. Using slightly lower resolution $(R=2000)$, comparable $S / N$ spectra, they give equivalent widths as small as $0.3 \AA$ reliably. Our upper limit on the strength of He II in IRAS 180791756 is smaller than this $(\sim 150 \mathrm{~m} \AA$ : we marginally detect a feature of this strength close to the expected He II wavelength, but it is compatible with the noise). Therefore, it is not possible to say definitively that IRAS 18079-1756 is not an embedded very late O-type photosphere. Nevertheless, since we do not detect He II with certainty, we adopt a spectral type of B2-B3, provided the luminosity class is nearer that of a dwarf than a supergiant, which seems likely.

As in the case of IRAS 17175-3544, IRAS 18079-1756 also shows a composite spectrum, with the $\mathrm{Al} \mathrm{I}$ and $\mathrm{Mg}$ I lines. The clear presence of a cluster around the latter object suggests that this spectrum may be composite for similar reasons to IRAS 17175-3544; namely the existence of a small cluster of unresolved low-mass embedded objects around or associated closely with the massive star. For IRAS 18079-1756, there is no suggestion of a second object in the slit which can be identified with a known object, unlike the case of IRAS 171753544 (IRS1-I 1W). The acquisition image PSF exhibits some asymmetry, but how many components it contains is unclear. Once again, we note that if the $\mathrm{MgI}$ and $\mathrm{AlI}$ lines are to be explained by a chance superposition of a late-type foreground object, there is no optical counterpart at the correct position on the SuperCosmos UKST I-band image.

\section{Discussion}

\subsection{The nature of the three sources}

Our sample of three embedded massive YSOs in UC H II regions has uncovered a variety of objects which may represent different stages of the earliest evolution of massive stars.
IRAS 17441-2910 shows broad, strong emission lines, and is known to show strong $\mathrm{CO}$ emission, indicating the presence of dense circumstellar material. While the intrinsic nearinfrared excess emission is marginal compared to that of IRAS 17175-3544, the presence of emission lines suggests mass accretion and/or outflow processes. This object appears to be at the earliest evolutionary phase of the three candidate massive YSOs.

IRAS $17175-3544$ is in some ways the most enigmatic source, since it has a flat continuum, but the largest near-IR excess of all. Its association with IRS-I 1 in the molecular cloud complex NGC 6334 (see Sect. 3) supports a young evolutionary status. The nebular emission detected ( $\mathrm{Br} 10, \mathrm{Br} 11$, $\mathrm{He} \mathrm{I}$ ) is narrower and weaker than in IRAS 17441-2910, but still clear. If the rather flat continuum of IRAS 17175-3544 is taken to mean it is relatively less embedded than IRAS 174412910 , the infrared excess is not explained. Instead, the weakness of the emission lines compared to IRAS 17441-2910 may be indirect evidence that IRAS 17175-3544 is less luminous than IRAS 17441-2910, and that the spectral type of the latter is earlier than O6-O7, which is supported by the previous derivation of a spectral type of O5.5 (see Table 1). Note however, that the broad emission in IRAS 17441-2910 is most likely of circumstellar rather than nebular origin. We propose that IRAS 17175-3544 may be at an equally early stage of evolution as IRAS 17441-2910.

Finally, the spectrum of IRAS 18079-1756 resembles a main sequence B-star, lacking direct spectral evidence for circumstellar material. The absence of near-infrared emission line features suggests a later evolutionary stage than the other two objects. However, the continuum does slope to the red, rising by $\sim 20 \%$ over the $750 \AA$ range covered by these spectra, similar to the slope exhibited by IRAS 17441-2910. Perhaps tellingly, IRAS 18079-1756 shows little near-infrared excess, while IRAS 17441-2910 does. This supports the hypothesis that IRAS 18079-1756 is more evolved but still obscured, possibly predominantly by intracluster dust.

While this would explain the slope of the continuum and the location of the source in Fig. 2, the association of the source with a UC $\mathrm{H}$ II region and $\mathrm{H}_{2} \mathrm{O}$ masering, and its identification as a CO outflow source by Osterloh et al. (1997), lend weight to the suggestion that it is an embedded (that is, still surrounded by circumstellar material) YSO.

We note also that the very presence of emission lines does not confirm these sources are massive embedded YSOs; OB supergiants such as P Cygni also exhibit emission lines in the near-infrared (Hanson et al. 1998), but such objects are not generally optically obscured, nor associated with masering phenomena.

\subsection{Spectral types from $H$-band data}

Where $\mathrm{H}$ and $\mathrm{He}$ absorption lines are detected, it is possible to obtain unambiguous spectral types, by comparison to galactic standards. He II absorption is detected in IRAS 17175-3544, in close quantitative agreement with the spectral type derived from IRAS fluxes, O6-O7. From the presence of strong He I 
and the absence of detectable He II in IRAS 18079-1756, we also confirm a spectral type of B3, with considerable accuracy ( $\pm \sim 3$ subclasses). These close agreements strongly suggest that near-infrared spectral types could be derived for many of the known UC H II regions for which far-infrared spectral types, derived from bolometric luminosities, are ambiguous (e.g. Walsh et al. 1997).

In a very recent paper, Hanson et al. (2002) have performed a large survey of UC HII regions in the $K$-band, including spectroscopy at $R=1200$. Their main finding relevant here is that $\sim 50 \%$ of their radio-selected sample were detectable by $\operatorname{Br} \gamma$ emission, but of those, only for $5-10 \%$ was it possible to detect photospheric features of the ionizing star, and thus derive spectral type and hence effective temperature directly. However, in the $K$-band spectroscopic survey of Kaper et al. (2002), at $R \sim 8000$, these authors detect characteristic $K$-band OB star features (emission and absorption) in the majority of their sample of 75 , of which $\sim \frac{1}{3}$ are found to be late-type foreground contaminants, and around 20 are found to be extremely strong $\mathrm{Br} \gamma$ emitters with obvious resemblances to IRAS 17441-2910, as discussed above. This suggests that if sufficiently high resolution and high signal-to-noise spectra $(R \geq 5000, S / N \geq 100)$ are used in either the $H$ or $K$ band, the photospheres of massive YSOs, at very early stages of evolution, could be readily detected, and absorption lines analysed. Such spectra would be amenable to similar techniques as those common in optical studies, in particular the derivation of gravities $\log g$ and projected rotational velocities $v \sin i$, both of which may be expected to differ systematically from that observed on the main sequence. Finally, given suitable line(s) present in the $H$-band, the detection of a surface enhancement of ${ }^{14} \mathrm{~N}$ in young massive stars suggests an observational test of the coalescence formation scenario, by analogy with O-type blue stragglers (Kendall et al. 1995).

\section{Conclusions}

1. We have used high signal-to-noise, intermediate resolution $H$-band spectroscopy to probe the central, ionizing stars of three UC H II regions. In two of the objects, IRAS 180791756 and IRAS 17175-3544, we detect the photosphere directly via absorption lines, allowing the derivation of spectral types which are in close agreement with those found by far-infrared observations.

2. In a third object, we detect no absorption, but a rich emission line spectrum. We identify this object, IRAS 174412910 , as an additional member of the small subset of massive YSOs displaying strong emission (including $\mathrm{CO}$ ) and which are likely to be at an extremely early evolutionary stage, surrounded by dense circumstellar material. The large infrared excess of IRAS 17175-3544, coupled with the observation of emission lines, also places it in this category.

3. IRAS 17175-3544 and IRAS 18079-1756 exhibit composite spectra, including absorption lines of neutral metals normally observed in cool stars, as well as $\mathrm{H}$ and $\mathrm{He}$ lines arising in the OB photospheres of or nebulosity associated with the targets themselves. Both these objects are associated with small clusters, as observed in our acquisition images. For IRAS 17175-3544, we identify the source of the metal lines as IRS-I 1W (Persi et al. 1996) and suggest that this object is a system of low mass embedded objects associated with the massive source itself. For IRAS 18079-1756 we tentatively suggest a similar scenario, although there is no direct evidence from imaging. In neither case can the spectra presented here rule out a chance superposition of a foreground late-type object causing the composite spectra, but if such a foreground star exists, it does not appear on SuperCosmos UKST I-band images of the two near-infrared sources.

4. $H$ or $K$-band spectra at a comparable or slightly higher resolution to those in this study, with high signal-to-noise ratio, may be amenable to quantitative analysis; e.g. derivation of projected rotational velocities and perhaps surface gravities, in the same way as for optical spectra. This will open up a new method to study massive YSOs at the earliest stages of their formation. Both bandpasses are likely to be useful: sources will be brightest in the $K$-band, where $\mathrm{CO}$ is also observed, but the $H$-band promises greatly reduced veiling and is likely to be a better wavelength region to seek weak metal absorption lines.

Acknowledgements. TRK and WJdeW acknowledge financial assistance from the European Union Research Training Network "The Formation and Evolution of Young Stellar Clusters" (RTN1-199900436). The authors thank the referee, Lex Kaper, for exhaustive comments and discussions. This publication makes use of data products from the Two Micron All Sky Survey, which is a joint project of the University of Massachusetts and the Infrared Processing and Analysis Center/California Institute of Technology, funded by the National Aeronautics and Space Administration and the National Science Foundation. This research has made use of the SIMBAD database, operated at CDS, Strasbourg, France

\section{References}

Amico, P., Cuby, J. G., Devillard, N., Jung, Y., \& Lidman, C. 2002, ISAAC Data Reduction Guide 1.5, ESO

Bonnell, I., Bate, M. R., \& Zinnecker, H. 1998, MNRAS, 298, 93

Bronfman, L., Nyman, L-Å., \& May, J. 1996, A\&AS, 115, 81

Campbell, B., Persson, S. E., \& Matthews, K. 1989, AJ, 98, 643

Caswell, J. L. 1998, MNRAS, 297, 215

Chan, S. J., Henning, Th., \& Schreyer, K. 1996, A\&AS, 115, 285

Dallier, R., Boisson, C., \& Joly, M. 1996, A\&AS, 116, 239

Emerson, J. P., Jennings, R. E., Moorwood, A. F. M. 1973, ApJ, 184, 401

Greene, T. P., \& Lada, C. J. 1996, AJ, 112, 2184

Hambly, N. C., Davenhall, A. C., Irwin, M. J., \& MacGillivray, H. T. 2001, MNRAS, 326, 1315

Hanson, M. M., Rieke, G. H., \& Luhman, K. L. 1998, AJ, 116, 1915

Hanson, M. M., Luhman, K. L., Rieke, G. H. 2002, ApJS, 138, 35

Harvey, P. M., \& Gatley, I. 1983, ApJ, 269, 613

Hughes, V. A., \& MacLeod, G. C. 1989, AJ, 97, 786

Ishii, M., Nagata, T., Sato, S., et al. 2001, AJ, 121, 3191

Kaper, L., Bik, A., Hanson, M. M., \& Coméron, F. 2002, in The Origins of Stars and Planets: The VLT View, ESO Astrophys. Symp. (Springer-Verlag)

Kendall, T. R., Lennon, D. J., Brown, P. J. F., \& Dufton, P. L. 1995, A\&A, 298, 489 
Kwok, S., Volk, K., \& Bidelman, W. P. 1997, ApJS, 112, 557

Lancon, A., \& Rocca-Volmerange, B. 1992, A\&AS, 96, 593

Luhman, K. L., \& Rieke, G. H. 1998, ApJ, 497, 354

Koornneef, J. 1983, A\&A, 128, 84

McBreen, B., Fazio, G. G., Stier, M., \& Wright, E. L. 1979, ApJ, 232, L183

Meyer, M. R., Edwards, S., Hinkle, K. H., \& Strom, S. E. 1998, ApJ, 508, 397

Morgan, J. A., \& Bally, J. 1991, ApJ, 372, 505

Neckel, T. 1978, A\&A, 69, 51

Osterloh, M., Henning, Th., \& Launhardt, R. 1997, ApJS, 110, 71

Persi, P., Roth, M., Tapia, M., et al. 1996, A\&A, 307, 591
Persson, S. E., \& Campbell, B. 1987, AJ, 94, 416

Porter, J. M., Drew, J. E., \& Lumsden, S. L. 1998, A\&A, 332, 999

Shu, F. H., Adams, F. C., \& Lizano, S. 1987, ARA\&A, 25, 23

Straw, S. M., Hyland, A. R., \& McGregor, P. J. 1989, ApJS, 69, 99

Tapia, M., Persi, P., \& Roth, M. 1996, A\&A, 316, 102

Walsh, A. J., Hyland, A. R., Robinson, G., \& Burton, M. G. 1997, MNRAS, 291, 261

Walsh, A. J., Burton, M. G., Hyland, A. R., \& Robinson, G. 1999, MNRAS, 309, 905

Watson, A. M., \& Hanson, M. M. 1997, ApJ, 490, L165

Wood, D. O. S., \& Churchwell, E. 1989, ApJ, 340, 265 